\title{
The use of histoacryl tissue adhesive for the primary closure of scalp wounds
}

\author{
R. J. MORTON, M. F. GIBSON \& J. P. SLOAN \\ Accident and Emergency Department, General Infirmary, Leeds, England
}

\section{SUMMARY}

A prospective evaluation of Histoacryl tissue adhesive for the repair of simple scalp음 wounds is described. Fifty wounds were repaired using the adhesive and, on examina-tion 5 days later, 49 wounds were fully healed, the remaining wound having only a small $\subseteq$ area of dehiscence. The advantage of this method of wound repair is discussed.

\section{INTRODUCTION}

Histoacryl is a tissue adhesive which is manufactured for use in closure of simple skin wounds ('Enbucrilate', B. Braun Medical Limited, Aylesbury, Bucks, UK). The butyl- $\overrightarrow{\overrightarrow{\vec{P}}}$ 2-cyanoacrylate monomer bonds tissue and can be used as an alternative or adjunct to 3 suturing. Although the adhesive has been available since 1959 (Coover et al., 1959), its use has been limited. This study was undertaken to assess the suitability of Histoacryl adhesive for primary closure of scalp wounds.

\section{PATIENTS, METHODS AND RESULTS}

Any patient attending the Accident Department of the Leeds General Infirmary, Leeds, England, aged between 4 and 75 years, with a scalp wound was considered for the trial. The following basic criteria were used for admission:

(1) Wound less than 6-h old

(2) Clean, incised wound

(3) Less than $6 \mathrm{~cm}$ in length

Correspondence: Dr R. Morton, Accident and Emergency Department, General Infirmary, Great George Street, Leeds LS1 3EX, England 
(4) Not broaching the galea

After clinical assessment, a skull X-ray was performed, if necessary, and the patient's informed consent for the procedure was obtained.

The wounds were cleaned, using a bactericidal cleaner, the tissue edges were opposed, using forceps, and a thin layer of adhesive applied. Tension was maintained for a minimum of $20 \mathrm{~s}$ to allow the edges to bond. The patient was instructed to keep the wound clean and dry. No dressings were applied. All wounds were reviewed 5 days later and assessed for any evidence of infection or dehiscence of the wound.

Fifty scalp wounds which satisfied the criteria were repaired with adhesive. Fortynine wounds were fully healed at 5 days without signs of infection. One $1 \mathrm{~cm}$ wound had $3 \mathrm{~mm}$ of dehiscence with some serous discharge, this required no further treatment. This single failure is not significantly different from an expected $100 \%$ success rate $(P<0.05$ Chi-squared test).

\section{DISCUSSION}

Suturing skin wounds is accepted as being an efficient method of obtaining skin closures. However, sutures have certain disadvantages. They are painful to insert and need to be removed at a later date. The residual scar may be marred by cross-hatching.

Histoacryl is a butyl-2-cyanoacrylate monomer, which forms a polymer when in contact with a small amount of water. The technique used (Kaplan, 1966) opposes the two moist edges of the wound. As the thin layer of adhesive is applied, a bond is formed. The histoacryl is coloured purple, to enable it to be accurately placed. The adhesive is biodegradable but remains until well after the wound has healed (Pam et al., 1968).

There are a few theoretical problems with the use of Histoacryl. The polymerization reaction releases heat which may cause tissue damage, though this is only very slight if a thin layer is applied. The heat may also cause some patient discomfort. The adhesive bonds rapidly, in less than $20 \mathrm{~s}$, therefore this does not allow for adjustment of the wound.

The advantages of the adhesive include speed and ease of application. The technique could be taught to anyone with the minimum of medical experience. The procedure is relatively painless and does not require local anaesthesia (this may be necessary to ensure adequate cleansing of the wound).

Return visits are unnecessary as there are no sutures to be removed. Fewer surgical instruments are used and sterilizing costs are reduced. The technique is eminently suitable for children. It can also be used in any superficial skin wound, for skin grafts (Jess et al., 1964) and for re-applying avulsed nails.

In this study, 50 scalp wounds were treated with adhesive. At review, only one wound had failed to achieve complete healing. Patient acceptability was high and the procedure rapidly gained popularity amongst the clinical staff.

The adhesive should be considered as an alternative to suturing small scalp wounds in an accident and emergency department. 


\section{REFERENCES}

Coover H. W. Jr, Joyner F. B., Shearer N. H. Jr \& Wicker T. H. Jr (1959) Chemistry and performance of cyanoacrylate adhesives. Special Technical Papers 5, 413-17.

Jess R. H. Jr, Andeson B. C. \& Healey J. E. Jr (1964) Fixation of split-thickness skin grafts with adhesive. Plastic and Reconstructive Surgery 33, 272.

Kaplan G. (1966) A technique of non-suture wound closure with a plastic tissue adhesive. Plastic and Reconstructive Surgery 73, 139-42.

Pam K. C., Gladieux G., Kulkarni R. H., Brandes G. \& Leonhard F. (1968) The degradation of N-butyl alpha cyanoacrylate tissue adhesive. Surgery 86, 481-9. 